(C) [2006] IEEE. Reprinted, with permission, from [Michael Ford, A new class of self-assembled monolayers on gold using an alkynyl group as a linker, Nanoscience and Nanotechnology, 2006. ICONN '06. International Conference on, 3-7 July 2006]. This material is posted here with permission of the IEEE. Such permission of the IEEE does not in any way imply IEEE endorsement of any of the University of Technology, Sydney's products or services. Internal or personal use of this material is permitted. However, permission to reprint/republish this material for advertising or promotional purposes or for creating new collective works for resale or redistribution must be obtained from the IEEE by writing to pubs-permissions@ieee.org. By choosing to view this document, you agree to all provisions of the copyright laws protecting it 


\title{
A new class of self-assembled monolayers on gold using an alkynyl group as a linker
}

\author{
M. J. Ford*, R. C. Hoft*, J. D. Gale ${ }^{1}$, A. M. McDonagh* \\ *Institute for Nanoscale Technology \\ University of Technology, Sydney, PO Box 123, Broadway NSW 2007, Australia \\ Email: mike.ford@uts.edu.au \\ ${ }^{1}$ Nanochemistry Research Institute \\ Curtin University of Technology, PO Box U1987, Perth, WA 6845, Australia
}

\begin{abstract}
The geometry and energetics for adsorption of ethynylbenzene on Au(111) have been studied using Density Functional Theory. The alkynyl group, following removal of the terminal $H$ atom, adsorbs covalently to the surface in the fcc hollow site with a bond energy of about $70 \mathrm{kcal}^{\mathrm{mol}}{ }^{-1}$. Intermediate adsorption states are also possible via a hydrogen 1,2 shift to form a surface-bound vinylidene, or through the opening of the $\mathrm{C}-\mathrm{C}$ triple bond without removing the hydrogen atom.
\end{abstract}

Keywords-component; self-assembled monolayers; density functional theory.

\section{INTRODUCTION}

Thiolate linkages are the most commonly used method for anchoring self-assembled monolayers to gold [1]. In the case of $\pi$-conjugated molecules it may be advantageous to link the conjugated moiety directly to the gold surface to provide an unbroken conjugation pathway. This may have considerable application to the study of transport properties and help resolve a number of open questions involving conduction in molecular wires. The alkynyl group is a potential candidate for such a linkage.

Metal-alkyne bonds have been studied extensively in the context of organometallic complexes [2], and a wide range of terminal alkynes are synthetically accessible. Two previous studies of ethynylbenzene adsorption on gold nanoparticles [3] and gold surfaces [4] using surface enhanced Raman spectroscopy appear in the literature. However, the nature of the bond is unclear from these works.

We have previously presented Density Functional Theory calculations of the adsorption of ethynylbenzene (Figure 1), a representative terminal alkyne, on a bulk $\mathrm{Au}(111)$ surface. The calculations allowed us to predict the adsorption energetics and potential pathways for forming self-assembled monolayers from this class of molecule [5].

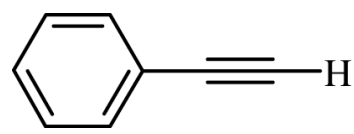

Figure 1. Ethynylbenzene
Here we extend these calculations to include a detailed mapping of the potential energy surface along the atop-bridgeatop path, and re-calculate interaction energies using a Zmatrix geometry optimization method.

\section{METHOD}

Calculations were performed using the SIESTA methodology and software [6]. This is a density functional, pseudopotential code with periodic boundary conditions. Atom centred basis sets describe the valence electrons. The key feature of SIESTA is that the orbitals are strictly localized in real-space, with a cut-off radius defined by a single energy shift parameter for all atoms that represents the energy increase in the orbitals due to confinement.

Basis set superposition errors (BSSE) have been corrected using the counterpoise method of Boys and Bernadi [7]. The generalised-gradient approximation (GGA) to the exchangecorrelation functional due to Perdew, Burke and Ernzerhof (PBE) [8] is used throughout. All calculations employ a Fermi smearing of $25 \mathrm{meV}$ to aid convergence. Core electrons are represented by norm-conserving pseudopotentials (with a relativistic correction in the case of $\mathrm{Au}$ ), and valence electrons by double-zeta plus single polarization basis sets.

Input atomic coordinates and geometry optimizations were carried out using a combination of internal and Cartesian coordinates. The gold atoms were specified by Cartesian coordinates and the adsorbate by a Z-matrix. Internal coordinates are more convenient for describing molecular geometries and can lead to increased efficiency during geometry optimization. We describe our implementation of internal coordinates in SIESTA elsewhere [9].

The gold substrate was modeled using a 4-layer slab for the potential energy scans and 7-layer slab for the final interaction energies, with $3 \times 3$ atoms in each layer. The lattice parameter is from an optimisation of bulk gold performed at the same computational level. The intermolecular spacing is greater than $0.5 \mathrm{~nm}$ and the intermolecular interaction, at this spacing, is calculated to be less than $1 \mathrm{kcal}^{\mathrm{mol}}{ }^{-1}$.

Convergence of the calculated energies with respect to the computational conditions, and transferability of the pseudopotentials have been checked carefully. At the highest computational parameters used here, interaction energies are 
converged to better than $1 \mathrm{kcal} \cdot \mathrm{mol}^{-1}$ [5]. At the intermediate level (the 4-layer calculations) a $3 \times 3 \times 1$ k-point grid in reciprocal space was used. Because the unit cell is quite large, a relatively coarse Monkhorst-Pack [10] mesh gives reasonably converged energies. The orbital energy shift was set to $5 \mathrm{mRy}$ and a mesh cutoff of 200 Ry was used for the real space integration of the density and related quantities. The mesh cutoff represents the highest energy plane-wave that can be represented on this grid. For the 7-layer calculations the kpoint grid was increased to $5 \times 5 \times 1$ and the real space grid to a cutoff of $250 \mathrm{Ry}$. In all calculations the force tolerances for geometry optimization were set to $0.04 \mathrm{eV} . \AA^{-1}$ for length and $0.0009 \mathrm{eV} . \mathrm{deg}^{-1}$ for angles. Although the length tolerance is not particularly tight, the bond lengths and interaction energies are not altered significantly even if this value is tightened to 0.01 $\mathrm{eV} . \AA^{-1}$

\section{RESULTS AND DISCUSSION}

The adsorption processes we are interested in studying result in a surface bound molecule in the configuration shown in Figure 2(a). Here, the terminal hydrogen (or proton) has been eliminated. There are a number of possible routes that can lead to this final product. The most direct is homo- or heterolytic cleavage of the $\mathrm{C}-\mathrm{H}$ bond followed by adsorption of the radical or anion. Intermediate surface bound states are also possible. Reactions of metals with ethynylbenzene can proceed via a 1,2 hydrogen shift to form metal vinylidenes [11]. The configuration of the corresponding surface-bound vinylidene is shown in Figure 2(b). The third possibility is via a $\pi$ interaction through both carbon atoms to give the flat geometry shown in Fig 2(c). The reaction could then proceed by $\mathrm{C}-\mathrm{H}$ bond cleavage (or deprotonation) to give the product shown in Fig 2(a).

The potential energy surface for the ethynylbenzene radical (configuration (a) in Fig 2.) moving along the path joining the atop-bridge-atop sites of the $\mathrm{Au}(111)$ surface has been calculated. Fig. 3 shows this path on the Au surface.

At each point in this potential energy scan the bonding $\mathrm{C}$ atom was fixed laterally relative to the slab and the remainder of the molecule and gold surface layer were allowed to relax. The relaxation was performed in two steps for each point; in the first stage the surface layer atoms and angle of the molecule relative to the surface were allowed to vary, and subsequently the remainder of the molecule. The use of internal coordinates for specifying the molecular geometry makes this kind of scan possible.

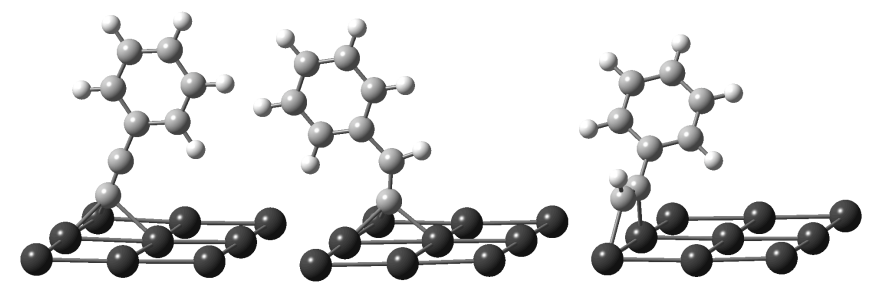

Figure 2. Configuration of surface bound molecule. (a) Final state, (b) vinylidene intermediate and (c) flat intermediate.

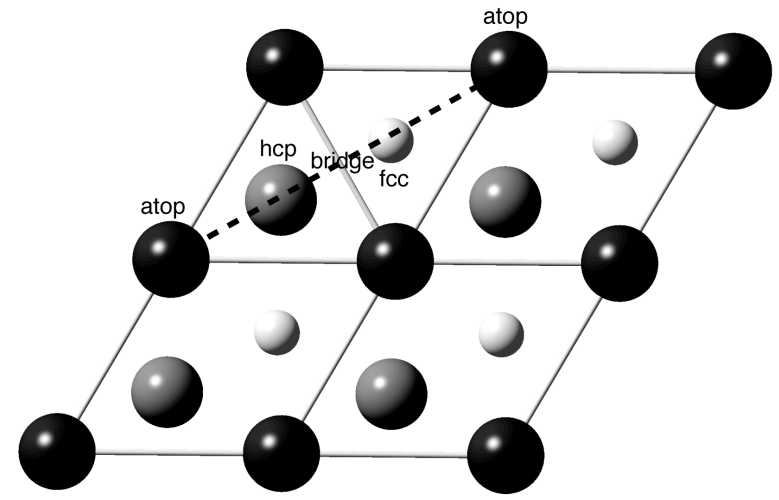

Figure 3. Path of potential energy scan on Au(111) surface (dotted line). View is normal to surface, first layer atoms are dark gray, second layer lighter gray and third layer white.

The energy along the potential surface in Fig. 4 is given relative to the minimum that occurs at the fcc hollow site. The hcp hollow is also a local minimum. The difference in total energy between these two sites is $1.8 \mathrm{kcal}_{\mathrm{mol}}{ }^{-1}$. The bridge site presents a barrier to diffusion between the two hollow sites of 3.2 and $1.4 \mathrm{kcal}^{\mathrm{mol}}{ }^{-1}$. This is an important quantity because the molecular headgroup must be relatively strongly bound to the surface yet sufficiently mobile that interactions between the tail groups can order the monolayer. In the case of ethynylbenzene the tail-tail interactions are likely to be dominated by $\pi-\pi$ interactions, which are of the order of 2.5 $\mathrm{kcal} . \mathrm{mol}^{-1}$ for a pair of molecules [12]. Hence, given the height of the diffusion barrier, we might expect this molecule to be able to form an ordered layer on gold. The optimized height of the $\mathrm{C}$ atom above the unrelaxed surface gold layer is also plotted in Fig. 4. As expected, the height of the molecule above the surface strongly correlates with the total energy.

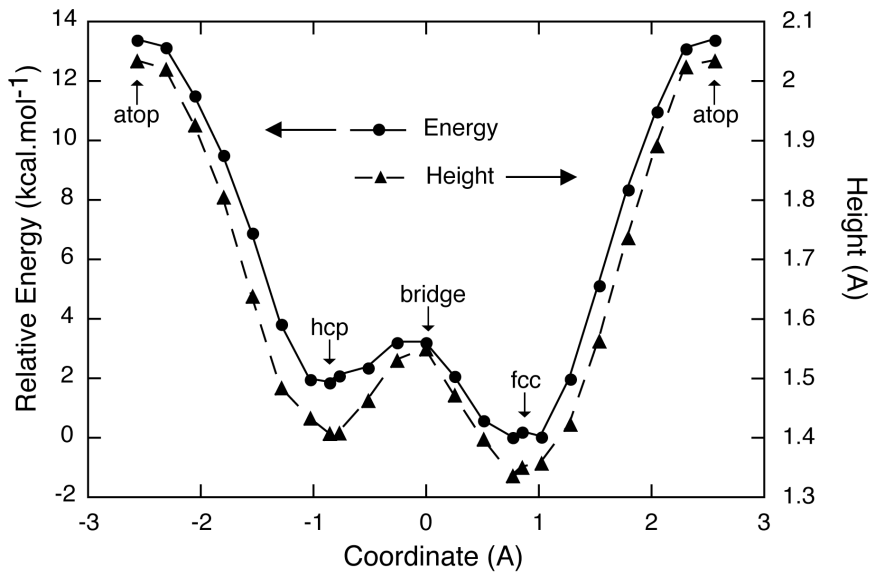

Figure 4. Potential energy scan for the ethynylbenzene radical along the atop-hcp-bridge-fcc-atop path. Coordinate is relative to bridge site. 
TABLE I. INTERACTION ENERGIES FOR THE ETHYNYLBENZENE RADICAL FINAL STATE (FIG 2(A)) VINYLIDENE (FIG 2(B)) AND FLAT MOLECULE (FIG 2(C)) INTERMEDIATE CONFIGURATIONS.

\begin{tabular}{llc}
\hline \hline Adsorbate & Site & $\begin{array}{c}\text { Interaction Energy } \\
\left(\mathrm{kcal}^{-\mathrm{mol}^{-1}}\right)\end{array}$ \\
\hline Ethynylbenzene & fcc & -68.9 \\
\hline Vinylidene & fcc & -53.8 \\
\hline Flat & atop & -43.5 \\
\hline \hline
\end{tabular}

We have also calculated the interaction energies for all three configurations given in Fig. 2 using our Z-matrix implementation. Geometries were initially fully optimized for a molecule and surface layer based on a 4-layer slab and intermediate computational parameters. Then final interaction energies were determined in a single-point calculation using these optimized geometries with a 7-layer slab and high-level computational parameters. The results are given in Table I. The final optimized geometries are shown in Fig. 2(a)-(c). Total Mulliken charges for the three adsorbates, overlap populations and bond lengths between terminal carbon atom(s) and nearest surface gold atoms are given in Table II.

After removal of the terminal hydrogen, the ethynylbenzene radical forms a strong chemisorption bond with the $\mathrm{Au}(111)$ surface with an interaction energy of -68.9 kcal.mol ${ }^{-1}$. The optimized geometry has the molecule tilted at $17^{\circ}$ to the surface normal, and the bonding carbon atom in the fcc hollow site shifted slightly towards the bridge. Hence, one of the bond-lengths to the nearest gold atoms is longer, by 0.1 $\AA$, and the corresponding overlap population smaller. The overall Mulliken charge of the surface bound ethynylbenzene radical and overlap population indicate a covalent bond forms with the gold surface. The corresponding quantities for the radical bound to a hydrogen atom are the same [5].

Using a Z-matrix optimization gives a slightly different final geometry compared with Cartesian coordinates in our previous work [5]. In the latter we found the molecule perpendicular to the surface, rather than tilted. The potential energy surface with respect to tilting the molecule relative to the surface is very flat. Internal coordinate optimization is more robust in this case because bond length optimization is de-coupled from angle optimization and, more importantly, different force tolerances can be used for each. The interaction energies predicted by the two methods are the same.

In contrast to thiol molecules, ethynylbenzene also forms a strong bond to gold without removal of the terminal hydrogen in the vinylidene (Fig. 2(b)) and flat (Fig. 2(c)) configurations as evidenced by the interaction energies in Table I. The vinylidene configuration adsorbs at the fcc hollow site shifted slightly towards the atop site (Fig 2.(b)). The bond lengths to the nearest three gold atoms are slightly smaller than in the ethynylbenzene case, but the overlap populations are almost twice as large. In addition, the bond to the surface appears to be more polar in character as the adsorbate charge is larger. In the flat geometry, the $\mathrm{C}-\mathrm{C}$ triple bond opens and two carbon atoms each bond to a surface gold atom. The optimized geometry has these two bonded carbon atoms close to atop sites (Fig. 2(c)).
TABLE II. MULLIKEN CHARGES, BOND LENGHTS AND OVERLAP POPULATIONS FOR THE THREE ADSORBATES.

\begin{tabular}{lccc}
\hline Adsorbate & $\begin{array}{c}\text { Adsorbate } \\
\text { Charge }\end{array}$ & $\begin{array}{c}\text { Bond } \\
\text { Lengths (A) }\end{array}$ & $\begin{array}{c}\text { Overlap } \\
\text { Populations }\end{array}$ \\
\hline \multirow{2}{*}{ Ethynylbenzene } & -0.147 & 2.298 & 0.126 \\
& & 2.194 & 0.189 \\
Vinylidene & -0.211 & 2.192 & 0.187 \\
\hline \multirow{2}{*}{ Flat } & & 2.170 & 0.243 \\
& -0.184 & 2.106 & 0.212 \\
\hline \hline
\end{tabular}

The results above suggest that both the vinylidene and flat configurations are possible intermediate states on route to the bound ethynylbenzene configuration. Direct formation of this final state requires a $\mathrm{C}-\mathrm{H}$ bond cleavage at considerable expense of energy. The pKa of ethynylbenzene is around 30. We have previously estimated the overall reaction energy, including bond cleavage and adsorption, to be endothermic to the order of more than $50 \mathrm{kcal}^{\mathrm{mol}} \mathrm{m}^{-1}$ [5]. Addition of a base during monolayer formation from solution may allow this reaction to proceed.

The overall reaction energy for formation of a surface-bound vinylidene and flat configuration are estimated to be exothermic or very slightly unfavourable [5]. Previous surface enhanced Raman (SERS) experiments suggest the possibility that ethynylbenzene can adsorb onto a gold surface in the flat geometry [4]. Furthermore, it is known that metal complexes with ethynylbenzene can be formed through deprotonation of the corresponding vinylidene [11].

Other reactions of the adsorbate in solution, or when exposed to air may also be possible. Recently, Deng and Friend have demonstrated experimentally that $\mathrm{Au}(111)$ can selectively oxidize styrene to the epoxide and the organic acid products benzoic acid and benzeneacetic acid [13]. However, these experiments were performed in vacuum with a gold surface previously dosed with atomic oxygen. Nonetheless, oxidation of the surface bound ethynylbenzene needs to be considered in SAM formation in solution.

\section{CONCLUSIONS}

We have performed density functional calculations using the generalised gradient approximation for single molecule adsorption of the ethynylbenzene radical on the $\mathrm{Au}(111)$ surface in a number of possible configurations. This work is motivated by the potential application of this molecule to form a new class of self-assembled monolayers.

We have implemented internal coordinate input and geometry optimization within the SIESTA code. This capability is still relatively unusual in periodic boundary conditions codes, and allows the molecule to be specified in the more convenient Z-matrix form and the gold slab in conventional Cartesian coordinates.

The calculations show that a strong covalent bond is formed with the surface upon removal of the terminal 
hydrogen. The fcc hollow site is most energetically favourable with an interaction energy of $68.9 \mathrm{kcal}^{\mathrm{mol}}{ }^{-1}$. The molecule can also undergo a hydrogen 1,2 shift and form a vinylidene surface-bound species, or adsorb flat on the surface by opening the triple bond and bonding through the two carbon atoms. The interaction energies in these two cases are calculated to be 53.8 and $43.5 \mathrm{kcal}^{\mathrm{mol}}{ }^{-1}$, respectively.

Using the Z-matrix format we have mapped the potential energy surface for diffusion of the ethynylbenzene adsorbate along the atop-hcp-bridge-fcc-atop reaction coordinate. The bridge site presents a diffusion barrier between the two hollow sites of less than $5 \mathrm{kcal}^{\mathrm{mol}} \mathrm{l}^{-1}$. This is the same order of magnitude as the $\pi-\pi$ interaction energy between the molecular tail groups that would drive self-assembly of the monolayer.

These calculations demonstrate that compounds incorporating the ethynylbenzene moiety offer a promising alternative to thiol compounds for anchoring organic molecules to gold surfaces to form self-assembled monolayers.

\section{ACKNOWLEDGMENT}

This work was supported by the Australian Research Council and the University of Technology, Sydney. AMM holds an Australian Research Council APD. JDG would like to thank the Government of Western Australia for support under the Premier's Research Fellowship Program. Computational resources were provided under the merit allocation schemes of ac3 (in NSW) and the National Facility, APAC.

\section{REFERENCES}

[1] J. C. Love, L. A. Estroff, J. K. Kriebel, G. Nuzzo, and G. M. Whitesides, "Self-assembled monolayers of thiolates on metals as a form of nanotechnology", Chem. Rev., vol. 105 pp. 1103, 2005.
[2] N. J. Long and C. K. Williams, "Metal alkynyl complexes: synthesis and material", Angew. Chem., vol. 42, pp. 2586, 2003.

[3] S.-W.Joo and K. Kim, "Adsorption of phenylacetylene on gold nanoparticle surfaces investigated by surface-enhanced Raman scattering", J. Raman Spectrosc., vol. 35, pp. 549, 2004.

[4] H. Feilchenfeld and M. J. Weaver, "Binding of alkynes to silver, gold and underpotential deposited silver electrodes as deduced by surfaceenhanced raman spectroscopy", J. Phys. Chem., vol. 93, pp. 42761989.

[5] M. J. Ford, R. C. Hoft, and A. M. McDonagh, "Theoretical study of ethynylbenzene adsorption on $\mathrm{Au}(111)$ and implications for a new class of self-assembled monolayer", J. Phys. Chem. B, vol. 109, pp. 20387, 2005.

[6] P. Ordejon, E. Artacho, and J. M. Soler, "Self-consistent order-n density-functional calculations for very large systems", Phys. Rev. B: Condens. Matter, vol. 53, pp. R10441, 1996; J. M. Soler, E. Artacho, J. D. Gale, A. Garcia, J. Junquera, and P. Ordejon, "The SIESTA method for ab initio order-n materials simulation", J. Phys.: Condens. Matter, vol. 14, pp 2745, 2002.

[7] S. B. Boys and F. Bernardi, "The calculation of small molecular interactions. Some procedures with reduced errors", Mol. Phys., vol. 19, pp. 533, 1970.

[8] J. P. Perdew, K. Burke, and M. Ernzerhof, "Generalized gradient approximation made simple", Phys. Rev. Lett., vol. 77, pp. 3865, 1996.

[9] R. C. Hoft, J. D. Gale, and M. J. Ford, "Implementation of a Z-matrix approach within the SIESTA periodic boundary conditions code and its application to surface adsorption", Mol. Sim, unpublished.

[10] Y. J. Wakatsuki, "Mechanistic aspects regarding teh formation of metal vinylidenes from allkynes and related reactions", Organomet. Chem., vol. 689, pp. 4092, 2004.

[11] H. J. Monkhurst and J. D. Pack, "Special points for Brillouin-zone integrations", Phys. Rev. B, vol. 13, pp. 5188, 1976.

[12] I. Dance, " $\pi-\pi$ interactions: theory and scope", in Encyclopedia of Supramolecular Chemistry, J. L Atwood, and J. W. Steed, Eds. New York: Dekker, 2004, pp. 1076.

[13] X. Deng and C. M. Friend, "Selective oxidation of styrene on an oxygen-covered $\mathrm{Au}(111)$ ”, J. Am. Chem. Soc., vol. 127, pp. 17178, 2005 . 